\title{
THE NATIONAL RADIO QUIET ZONE AND THE GREEN BANK RFI ENVIRONMENT
}

WESLEY A. SIZEMORE

National Radio Astronomy Observatory 1

Green Bank, West Virginia 24944

\section{INTRODUCTION}

Today, I would like to discuss with you the history of the National Radio Quiet Zone, the regulation procedures for fixed radio transmitters inside the NRQZ and the RFI environment at the National Radio Astronomy Observatory (NRAO) site in Green Bank, West Virginia.

\section{ESTABLISHMENT OF THE NRQZ}

The NRQZ was established in the National Telecommunications and Information Administration's (NTIA) Interdepartment Radio Advisory Committee (IRAC) Document 3867/2 dated 26 March 1958 and Federal Communications Commission (FCC) Rule Making Document Number 11745 dated 19 November 1958 and encloses an area of approximately 34,000 square kilometers of the States of Virginia and West Virginia. The purpose of the NRQZ is "... to minimize harmful interference at the NRAO site located at Green Bank, Pocahontas County, West Virginia, and at the Naval Research Observatory at Sugar Grove, Pendleton County, West Virginia." [1] and provides interference protection beyond that provided by the frequency allocation process.

\section{REGULATION BODIES}

The bodies having approval authority for transmitters inside the NRQZ are the FCC for non-Government applications and the National Telecommunications and Information Administration for Government applications.

A. FCC Rules and Regulations state that "Any applicant for a new permanent base or fixed station, or for modification for an existing authorization which would change the frequency, power, antenna height, directivity or location

1. The National Radio Astronomy Observatory is operated by Associated Universities, Inc. under contract with the National Foundation 
within the boundaries ... of the NRQZ ... shall notify the Director, National Radio Astronomy Observatory, P. O. Box 2, Green Bank, WV 24944, in writing, of the technical parameters of the proposal." Upon receipt of this notification, the NRAO has a period of 20 days in which to comment or file an objection in response to the notification. The FCC then considers all aspects of the problem and takes whatever action is deemed appropriate. Heretofore, the FCC has strongly supported the NRQZ.

B. The NTIA Manual states that "... in order to minimize harmful interference to operations at the ..." NRAO site in Green Bank, WV, and the Navy research station at Sugar Grove, WV, "... all requests for frequency assignments to stations within the ... [NRQZ] ... shall be coordinated by the applicant, prior to authorization with ..." NRAO.

\section{EXPLANATION OF PROPAGATION ANALYSIS}

Upon notification of an application being filed for a new or modification to an existing station, a propagation analysis is conducted to determine the maximum allowable effective radiated power (ERP) in the direction of Green Bank that a radio transmitter in the NRQZ may have and still comply with the power density limits established for the protection of the NRAO. More on power density limits will be discussed.

In order to perform a propagation analysis, the following information is required.

1. Antenna location in latitude and longitude to nearest second.

2. Antenna site ground elevation above mean sea level (AMSL).

3. Antenna (radiation center) height above ground level (AGL).

4. Frequency or frequency range of each transmitter.

When this information is received, the coordinates are plotted on a U.S. Geological Survey 7.5 minute series topographic map to insure that the ground elevation and antenna location are correct. If the information provided is correct, then the data are entered into a computer program based on National Bureau of Standards Technical Note No. 101 entitled "Transmission Loss Predictions for Tropospheric Communication Circuits" by Rice, Longley, Norton and Barsis. The program computes the following parameters:

1. True azimuth bearing from receiver (NRAO) to transmitter.

2. True azimuth bearing from transmitter to receiver.

3. Distance between transmitter and receiver.

4. Receiver and transmitter site elevations.

5. Elevation, radius, and distance of obstacles in the radio signal path from the transmitter to the receiver.

The above distances and elevations are obtained from a digitized topographic map of the NRQZ. Once the above parameters are computed, the program calculates the total diffraction attenuation, troposcatter attenuation, and free space attenuation for the specified frequency to arrive at a total path attenuation. From the total path attenuation, frequency and the NRAO limit for received signal power density, the program computes the maximum allowable 
ERP relative to a dipole on a true azimuth bearing from the transmitter to the receiver reference point at NRAO, Green Bank, West Virginia.

\section{POWER DENSITY LIMITS}

The NRAO has established power density limits which, if exceeded by new transmitters or modifications to existing transmitters, could result in substantial degradation in the RFI environment at the NRAO, Green Bank.

Any radiation in a primary radio astronomy band is considered harmful. In other radio astronomy bands and narrow guard bands adjacent to all radio astronomy bands, the limit is set to the CCIR Report 224-4 level. At all other frequencies the limits were set by demonstrable receiver sensitivities, standards for antenna sidelobe responses (CCIR Report 224-5), proximity of the frequency to a protected radio astronomy band and existing interference conditions over which the NRAO has no control, e.g., transmitters located outside the NRQZ.

\section{RFI ENVIRONMENT AT GREEN BANK}

The RFI environment at the NRAO site in Green Bank, WV, is very favorable for radio astronomy. The NRQZ provides substantial protection from interference from fixed radio transmitters and the rural location, as well as natural terrain shielding, provide additional protection. However, like any site, some degree of interference is present from unintentional as well as intentional RF emitters.

A. Unintentional RF emitters fall within two main categories controllable and uncontrollable sources:

1. By controllable sources, I mean such sources as faulty power transmission lines, oscillating TV signal amplifiers and defective electrical switches and thermostats in the vicinity of the NRAO as well as signals generated on the NRAO site. This type of interference is located by using mobile RFI monitoring equipment. Once the faulty equipment is located, the necessary steps to correct the problem are taken such as notifying the local power company of power line problems and replacing or repairing defective switches or TV signal amplifiers.

2. Uncontrollable sources of RFI such as lightning are avoided whenever possible by adjusting observing schedules or reduced in severity by various data manipulation techniques.

B. Intentional RF emitters are placed into four main categories:

1. RFI from fixed transmitters inside the NRQZ, both Government and non-Government, is maintained at an acceptable level by the NRQZ. However, some exceptions to the power density limits established for the NRQZ have been made. The NRAO is concerned about public safety and works out cooperative agreements with the relevant agencies, such as police, that respond to emergencies. Exceptions to the power density limits are rare and granted only after all other possible solutions are exhausted. Also, there are some transmitters 
that were authorized before the NRQZ was established and thus are "grandfathered".

2. Mobile transmitters in the NRQZ include both Government and nonGovernment, surface and airborne users. However, this potential source of RFI is minimal due to the rural location of the Observatory and the lack of any major Government installation in the area. Additionally, several military commands that use the airspace over the NRAO are notified monthly of our observing schedule and their voluntary cooperation in reducing RFI is solicited. This arrangement has worked very well. The various military commands have, without exception, been extremely cooperative in limiting potentially interfering transmissions when operating in the vicinity of the Observatory.

3. Fixed and mobile transmitters outside the NRQZ generate some detrimental RFI, but because of the size of the NRQZ and the Observatory's location within it and shielding provided by the mountainous terrain, these potential sources of interference are also minimal.

4. The last category of international RF emitters is satellites. I am sure we are all aware of the severe RFI problems from the GLONASS satellites, and I fear that the satellite RFI situation as a whole will continue to worsen. In order to gain some control over the satellite RFI situation, the NRAO is in the process of building a data base of pertinent satellite data such as unclassified transmit frequencies and orbital elements for most satellites from the National Aeronautics and Space Administration (NASA) but obtaining a complete list of unclassified satellite transmit frequencies has proven to be a most difficult problem. The frequency information we now have in the data base is obtained from commercial and scientific publication. The only comprehensive list of unclassified satellite transmit frequencies that I am aware of is the Federal Government's Master List and access to this data has been denied.

Due to the international nature of the satellite RFI situation, a comprehensive satellite data base may best be constructed and made available to the radio astronomy community by an international organization such as the Inter-Union Commission on Frequency Allocations for Radio Astronomy and Space Science (IUCAF). Therefore, I suggest that the IUCAF study the feasibility of establishing a comprehensive satellite data base that would be accessible by the entire radio astronomy community.

\section{CONCLUSION}

In conclusion, I would like to reiterate the two main reasons for this presentation: First, the National Radio Quiet Zone was established for the purpose of providing additional interference protection to radio astronomy beyond that provided by the frequency allocation process and it is fulfilling its purpose. Thus, the National Radio Astronomy Observatory in Green Bank, WV, is afforded RFI protection beyond that provided to any other Observatory. That is not to say that Green Bank does not experience RFI, only that the RFI environment is good by today's standards.

The other main point I wish to make is the seriousness of the satellite RFI situation. I am aware that many people are working on this problem and on several fronts, but I believe that one of the first steps should be the establishment of a satellite data base that is accessible by all. Due to the international nature of 
the problem, I feel an organization such as the IUCAF may be better equipped for this task, and I urge the IUCAF to investigate the feasibility of such a data base.

\section{REFERENCE}

[1] Federal Communications Docket No. 11745 adopted November 19, 1958. 